\title{
VISUAL OUTCOME OF PHACOEMULSIFICATION WITH THE HYDROPHILIC IOL IMPLANTATION IN COMPLICATED CATARACT SECONDARY TO UVEITIS
}

\author{
Jayshri V. Ekhar', Mona Deshmukh², Ashok H. Madan³ \\ ${ }_{1}^{1}$ Assistant Professor, Department of Ophthalmology, Government Medical College, Nagpur. \\ ${ }^{2}$ Associate Professor, Department of Ophthalmology, Government Medical College, Nagpur. \\ 3 Professor \& HOD, Department of Ophthalmology, Government Medical College, Nagpur.
}

ABSTRACT: AIM: To study the Visual outcome of Phacoemulsification with the hydrophilic IOL implantation in complicated cataract secondary to uveitis.

METHOD: This was a prospective study on 40 eyes of 40 patients with uveitic cataract, who underwent Phacoemulsification with hydrophilic IOL at a tertiary care centre in central India over a period of 2 years. In our study after preoperative control of inflammation for minimum 3 months Phacoemulsification was performed and results were observed with the aim to study visual outcome of Phacoemulsification with hydrophilic IOL in complicated cataract secondary to uveitis.

RESULTS: Mean age of the subjects in the study was 40.5 yrs. Etiologically, idiopathic uveitis was most common cause (24 cases), followed by tuberculosis ( 8 cases). One year post-operatively, $92.5 \%$ of subjects had $\geq 2$ snellen's lines improvement and of these $80 \%$ (30) of subjects had visual acuity of $\geq 6 / 18$. The main complications affecting visual outcomes were Macular involvement and optic neuropathy. PCO was the commonest complication observed in 12 subjects.

CONCLUSION: Phacoemulsification with implantation of a hydrophillic PC IOL in the capsular bag is safe and effective in patients with coexisting uveitis and cataract, provided that the ocular inflammation is under control.

KEYWORDS: Complicated cataract, Uveitis, Phacoemulsification.

HOW TO CITE THIS ARTICLE: Jayshri V. Ekhar, Mona Deshmukh, Ashok H. Madan. "Visual Outcome of Phacoemulsification with the Hydrophilic IOL Implantation in Complicated Cataract Secondary to Uveitis." Journal of Evolution of Medical and Dental Sciences 2015; Vol. 4, Issue 94, November 23; Page: 15999-16001, DOI: 10.14260/jemds/2015/2332.

INTRODUCTION: Cataract is a common complication of longstanding uveitis and chronic corticosteroid therapy. It is mostly posterior subcapsular in location. Incidence of cataract varies with etiology. Apart from cataract, other causes of low vision in uveitis are: Chronic macular oedema, Epiretinal membrane, Optic neuropathy and glaucoma. Cataract surgery is often indicated for visual rehabilitation and to allow assessment and management of posterior segment pathology. Phacoemulsification with posterior chamber intraocular lens (PC IOL) implantation is the preferred cataract surgery technique in patients with uveitis.

The outcomes depend on the cause and severity of uveitis as well as preoperative and postoperative control of the inflammation. In our study after preoperative control of inflammation for minimum 3 months Phacoemulsification was performed and results were observed with the aim to study visual outcome of Phacoemulsification with hydrophilic IOL in complicated cataract secondary to uveitis.

STUDY DESIGN: This was a prospective study on 40 eyes of 40 patients with uveitic cataract, who underwent Phacoemulsification with hydrophilic IOL at a tertiary care centre in Central India over a period of 2 years. Retinal detachment, vitreous haemorrhage or herpetic keratoconjunctivitis. Phacoemulsification with PC-IOL implantation were included in study.

Financial or Other, Competing Interest: None.

Submission 06-11-2015, Peer Review 07-11-2015,

Acceptance 13-11-2015, Published 23-11-2015.

Corresponding Author:

Dr. Jayshri V. Ekhar,

Flat No. 201, B-wing,

Poonam Heights, Pande Layout, Khamla,

Nagpur-440025, Maharashtra.

E-mail: vipinekhar@yahoo.com

DOI:10.14260/jemds/2015/2332.
All patients who presented to our OPD having chronic uveitis with visually significant cataract and who underwent Phacoemulsification with PC-IOL implantation were included in study. Pre-operatively the inflammation was kept under control for a minimum of 3 months and only those cases having minimum postoperative followup of 6 months were considered for the study. Exclusion criteria were active inflammation, subjects requiring combined procedure (Phacoemulsification with trabeculectomy) and subjects having diminution of vision because of other aetiologies like retinal detachment, vitreous haemorrhage or herpetic keratoconjunctivitis.

Pre-operative Work-up: At initial visit demographic data was recorded, detailed history with regards to the type and duration of symptoms and any associated co-morbidities were noted. A through general examination and ocular examination was done. Ocular examination included: Snellen's visual acuity (Both eyes), slit lamp examination, pupillary size with dilatation, posterior synechiae documentation, cataract grading, IOP measurement by Goldmann applanation tonometer, gonioscopy and fundus examination. Blood investigations like blood sugar, complete blood count, RA factor, C-reactive protein, erythrocyte sedimentation rate, antinuclear antibody levels, mantoux test, FTA-ABS test/VDRL, ELISA-HIV test were performed in every case to ruleout certain specific causes of uveitis.

Radiological investigations included chest x-ray, x-ray sacroiliac joint and B-scan if posterior details not visualised by ophthalmoscopy. Other investigations like skin biopsy, urine test for WBCs were done as and when indicated.

Pre-operative Treatment: All patients received oral prednisone in the dose of $1 \mathrm{mg} / \mathrm{kg}$ body weight in tapering doses with $1 \%$ Prednisolone eyedrops and 1\% atropine eyedrops for 15 days pre-operatively. 
PROCEDURE: Surgical procedure was performed by a single surgeon under local anaesthesia. Manual pupillary dilatation was done by iris hooks when required. Synechiolysis was done by lens dialer and viscoelastic substances. In cases where synechiae were not present, BSS with adrenaline was injected. Pupillary dilatation was ensured in all cases. Capsule was stained and capsulorhexis was done with 26 no. Cystitome. CCC of adequate size and well centered was performed to complete nuclear fragment removal and overlapping of optic of IOL. Phacoemulsification was done by divide and conquer method. Hydrophilic (Acrylic) IOL was injected and properly placed in capsular bag. Thorough anterior chamber wash was given. Subconjunctival injection of Dexamethasone and Gentamycin was given in immediate post-operative period.

Post-operative Treatment: Systemic steroids in the form of oral Prednisolone in the dose of $1 \mathrm{mg} / \mathrm{kg}$ body weight in tapering doses with 1\% Prednisolone suspension eye drops was instilled every half hourly for first 24 hours followed by 1 hourly for another 2 days and then tapering in 4-6 weeks; $1 \%$ atropine eye drop was given as cycloplegic-mydriatic drug. Post-operative visual acuity was recorded on day 1 , after 1 week, after 6 months and the end of 1 year. Complications were recorded and treated as and when needed.

Observations and Results: Mean age of the subjects in the study was 40.5 yrs. with age range of $21-60$ yrs; $55.5 \%$ subjects were women and $45.5 \%$ were men. Etiologically, idiopathic uveitis was most common cause (24 cases), followed by tuberculosis ( 8 cases), Fuchs' heterochromic iridocyclitis ( 3 cases) sarcoidosis (2 cases), leprosy (2 cases), scleronegative spondyloarthropathy (1 case) in that order. Pre-operatively, the visual acuity in all patients ranged from PL+ and PR acurate to 2/60. Post-operatively, at first visit (One week post-operative) $83 \%$ of subjects had $\geq 2$ Snellen's lines improvement in visual acuity. Of these, majority of subjects i.e. $74.3 \%$, had visual acuity of $\geq 6 / 18$.

At last visit (One year post-operatively), the subjects with $\geq 2$ Snellen's lines improvement increased to $92.5 \%$ and of these $80 \%$ (30) of subjects had visual acuity of $\geq 6 / 18$. The main complications affecting visual outcomes were macular involvement and optic neuropathy. CME (4 cases) and secondary glaucoma ( 2 cases) had poor visual outcome. PCO was the commonest complication observed in 12 subjects. It was treated with Nd YAG capsulotomy under pre and post procedure steroid cover. Other complications included Posterior synechias (8), Iris bombe (4), Uveitis recurrence (2), Epi-retinal membrane (1), Membrane over IOL (1), IOL decentration in capsular bag (1), Hypopyon (1) and fibrinous reaction (1).

DISCUSSION: Cataract formation is the most common cause of vision loss in patients with uveitis. Successful surgical treatment is essential for optimum visual rehabilitation and adequate visualization of the posterior segment. However, the cataract surgery in uveitis patients is challenging for the following reasons. ${ }^{1}$

1. Associated Iris pathologies like posterior synechiae with small constricted pupil.

2. Presence of pupillary membrane.

3. Permanent breakdown of blood-aqueous barrier.

4. Propensity of exuberant post-operative inflammatory reactions.
To overcome above challenges, Phacoemulsification with foldable IOL is preferred procedure in such cases. ${ }^{1}$ The advantages of this procedure. ${ }^{2}$ are:

1. Small incision surgery (In avascular cornea).

2. Reduced iris manipulations.

3. Decreased post-operative inflammation.

4. Faster healing and recovery.

In our series, successful phacoemulsification was completed in all cases and the IOL was implanted in the capsular bag except in 1 eye, in which posterior capsule rupture occurred during removal of the cortex. In this case, a 2-port limbal anterior vitrectomy was performed and the IOL implanted in the ciliary sulcus.

The causes of uveitis are many, idiopathic cause being the most common. In our study, idiopathic cause was seen in 24 cases followed by presumed tuberculosis (8 cases); other causes included Fuchs' heterochromic iridocyclitis (3 cases), sarcoidosis (2 cases), leprosy (2 cases) and scleronegative spondyloarthropathy ( 1 case). This observation is in accordance with the literature. In a study by Singh et al.,3 tuberculosis was responsible for $69.8 \%$ of infective uveitis cases.

Phacoemulsification with IOL implantation improved vision in most patients with coexisting cataract and uveitis. ${ }^{4}$ In our study, postoperatively, the corrected distant visual acuity (CDVA) improved to $6 / 18$ or better in 30 eyes (75\%) and $6 / 24$ in 4 eyes (10\%). Visual acuity did not improve in the remaining 6 eyes $(15 \%)$ despite successful surgical treatment because of optic atrophy or macular degeneration with scar formation. Our results are comparable to those of previous studies of cataract surgery in eyes with uveitis. In a study by Rauz et al.,5 73.3\% of eyes achieved a visual acuity of $20 / 30$ or better after phacoemulsification with foldable PC IOL implantation. However, our visual outcomes were better than those in a study by Alio's et al., 6 in which only $46.3 \%$ of eyes achieved a visual acuity of 20/40 or better.

As regards to complications, vision affecting complications were mostly related to macular degeneration and secondary optic atrophy. These complications were observed in 6 subjects in our study and were responsible for poor outcome in $15 \%$ of our subjects despite a successful surgery. PCO is the commonest of the complications, encountered in 12 eyes (30\%); however, it was treated with Nd YAG laser capsulotomy and did not have a permanent effect on visual acuity. These findings are in accordance with study of Rauz et al. ${ }^{5}$ However, Alio et al., 6 in their study found vision threatening complications to be more common.

CONCLUSION: In conclusion, phacoemulsification with implantation of a hydrophillic PC IOL in the capsular bag is safe and effective in patients with coexisting uveitis and cataract, provided that the ocular inflammation is under control. Identifying patients who are at risk of postoperative complications helps in patient counselling and to pre-empt these complications by using preoperative prophylactic corticosteroids, careful IOL selection and postoperative intensive corticosteroids.

To minimize vision-limiting complications, these patients must be observed closely after surgery and must be treated aggressively if the inflammation recurs. 
Thus with careful patient selection, appropriate preoperative preparation, diligent surgery and close postoperative supervision, phacoemulsification with intraocular lens implantation is safe and effective in the great majority of eyes with uveitis.

\section{REFERENCES:}

1. Suresh P.S. and Jones. N.P. Phacoemulsification with intraocular lens implantation in patients with uveitis. Eye (2001); 15:621-628.

2. Jagat Ram, Amod Gupta, Sunil Kumar, Sushmita Kaushik, Nishant Gupta, Sourabh Severia. Phacoemulsification with intraocular lens implantation in patients with uveitis. J Cataract Refract Surg 2010; 36:1283-1288.
3. Singh R, Gupta V, Gupta A. Pattern of uveitis in a referral eye clinic in north India. Indian J Ophthalmol 2004; 52:121-125.

4. Okhravi N, Lightman SL, Towler HMA. Assessment of visual outcome after cataract surgery in patients with uveitis. Ophthalmology.1999; 106:710-722.

5. Rauz S, Stavrou P, Murray PI. Evaluation of foldable intraocular lenses in patients with uveitis. Ophthalmology 2000; 107:909-19.

6. Alio' JL, Chipont E, BenEzra D, Fakhry MA. Comparative performance of intraocular lenses in eyes with cataract and uveitis; the International Ocular Inflammation Society Study Group of Uveitic Cataract Surgery. J Cataract Refract Surg 2002; 28:2096-2108. 